\title{
BAND TO BAND REGISTRATION OF MULTI-SPECTRAL AERIAL IMAGERY - RELIEF DISPLACEMENT AND MISS-REGISTRATION ERROR
}

\author{
M. Hassanpour ${ }^{1}$, F. Dadras Javan ${ }^{1, *}$, A. Azizi ${ }^{1}$ \\ ${ }^{1}$ School of Surveying and Geospatial Engineering, University College of Engineering, University of Tehran, Tehran, Iran - \\ (fdadrasjavan, mhd.hassanpour)@ut.ac.ir
}

Commission VI, WG VI/4

KEY WORDS: Band to Band Registration, Multi-Spectral Imagery, Relief Displacement, Miss-registration Error, Aerial Imagery

\begin{abstract}
:
With the development of UAVs in agricultural applications, multi-spectral cameras are designed to be installed on these platforms in small size and weight to help users for applications. These cameras are designed in multi lenses structure each lens considered for each spectral band. Band miss-registration of low altitude multispectral images acquired via UAVs is challenging due to multi view image sensors and optics structure of the multispectral cameras. In this study, an image based registration method is proposed to reduce the effect of relief displacement on miss-registration error. For this purpose, three different data sets with different level of relief displacement are considered and three different strategies are proposed. In the proposed method, by dividing the image into patches, selecting the appropriate local window, and local matching in each window, an attempt has been made to obtain the proper distribution of points in the image. Also, by limiting the search space, the matching speed is also increased. To evaluate the proposed method, the comparison with the ENVI and ArcGIS software has been made where results clearly present the superiority of the proposed method by average of 0.28 pixel in accuracy.
\end{abstract}

\section{INTRODUCTION}

In image processing applications, image registration is one of the most important demands (Aicardi, et al, 2016). Alignment of images taken from UAVs, airplanes, or satellites, image registration is highly needed (Pritt, et al, 2014). The visible cameras usually have 3 bands including Red, Green, and Blue bands, where they are not much applicable in the field of agriculture and vegetation based application (Zhang, et al, 2016). For this propose, multi-spectral cameras that have more than 3 visible bands are used, which can be noted the near infrared or red edge bands beside the visible bands. Multi-spectral images are usually captured by satellites, which later evolves with the development of technology and UAVs, multi-spectral cameras are built in small size to be installed and operated on the UAVs (Grant, 2017). For example, Red-Edge camera (MicaSense, Inc., USA), which has five bands of Red, Green, Blue, Near Infrared, and Red-edge which structure is presented in figure 1.

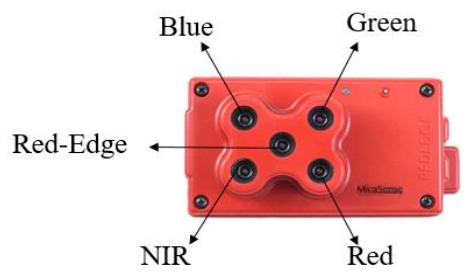

Figure1. Red-Edge camera structure

In multi-spectral images that captured by UAVs and airplanes, because of the frame based geometry and the multi lens structure of these sensors, band to band registration is critical before using these images in further processing application (Zhao, et al, 2018). In this context, there are two approaches for image registration as object level and image level. In the image based strategy, all the steps of registration are done in image space. Xiaoyang Zhao (2018), registered bands of Red-Edge's camera through local window, Fang Xu (2018) for multi temporal image registration used two steps of coarse registration and fine registration, Yanjia chen (2017) for visible and infrared images registration applied two steps of coarse and fine registration. But in the object based strategy, images are registered in the object space or the model space. Hao Gan (2018), for visible and thermal camera registration, and Jyun-Ping Jhan (2016-2017-2018) for band to band registration of mini-MCA's camera used object based approaches.

When multi-spectral cameras are installed on UAVs and image are captured at low altitudes, miss registration error between bands becomes more distorted. Also, in the situation of high elevation differences, the error resulting from the relief displacement appears in the images which presented in figure 2.

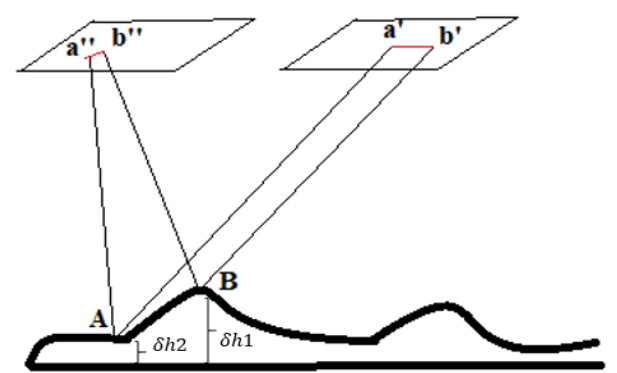

Figure2. Relief displacement

Accordingly, the following points can be considered:

- The imaging height is low in many applications, such as agriculture, with the difference in the height of the areas, and the relief displacement is unavoidable.

- This difference has been made in applications such as diagnosis of pests, plant diseases, and so on where even a pixel displacement may

\footnotetext{
* Corresponding author
} 
- $\quad$ lead to false conclusion. It is necessary to correct this error.

- $\quad$ Multi-spectral cameras include multi lenses. The 5-lens Red-Edge camera is one of the most common cameras used in agricultural applications.

The purpose of this study is to develop a new method for band to band registration of aerial multi-spectral images which is composed of choosing a suitable feature points extractor, selecting the optimal solution for data with different features and different height displacement, and if necessary, selecting a local window to detect feature points with proper distribution throughout the images. Finally, for accuracy assessment of the study, results are compared to those achieved by ENVI and ArcGIS software.

\section{MATERIALS AND METHODS}

\subsection{Test Images}

Test images are taken by Red-Edge camera (MicaSense, Inc., USA), which has five spectral bands including Red, Green, Blue, Near Infrared (NIR), and Red-Edge by a small multirotor platform, over 3 different height relief fields. These areas cover a flat area (dataset 1) (micasense.com), the cultivating area with bushes with moderate height difference (dataset 2) (micasense.com), and a Tree Garden area with trees of varying heights (dataset3). For dataset 3, some of flight planning information as follows:

Flight altitude is $25(\mathrm{~m})$, Ground resolution is 12.7 ( $\mathrm{mm} / \mathrm{pix})$, Footprint is $16.25 \times 12.45(\mathrm{~m} 2)$.

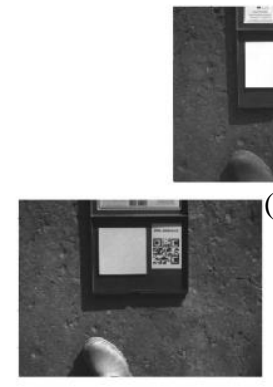

(c)
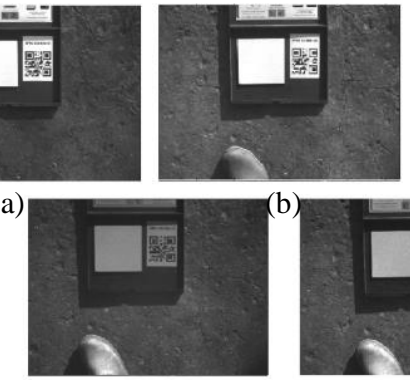

(d)

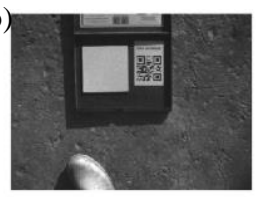

(e)
Figure 3. Data set 1: the image of a calibration panel in five bands with almost flat contents: (a) red, (b) green, (c) blue, (d) NIR, (e) red-edge.

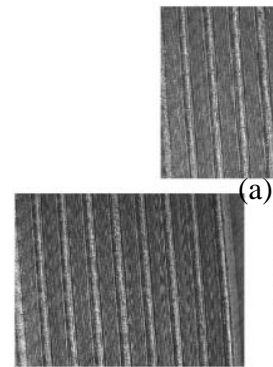

(c)

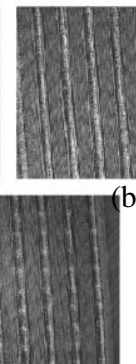

(d)

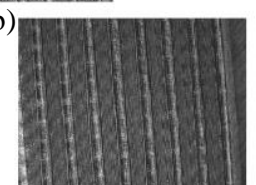

(e)
Figure 4. Data set 2: the image of an agricultural area in five bands with contents of mid height relief: (a) red, (b) green, (c) blue, (d) NIR, (e) red-edge.
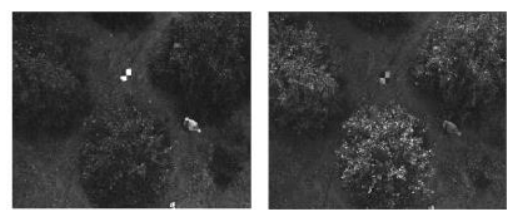

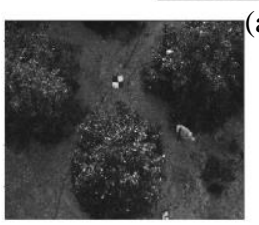

(c) (d)

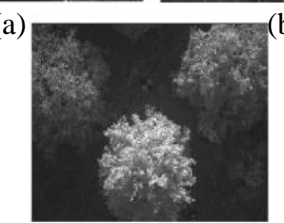

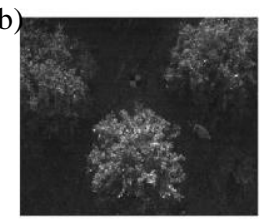

(e)
Figure 5. Data set 3: the image of an orchard in five bands with high altitudes differences, up to 6 meters: (a) red, (b) green, (c) blue, (d) NIR, (e) red-edge.

\subsection{Proposed Band to Bands Registration's Methods}

Due to the optics and lenses structure of the multi-spectral camera, the images of this type of camera do not align with each other, and if the images are taken at low altitude, an error due to the lack of uniformity of the height difference displacement in the images is created. For a better understanding, this is shown in Figures 6, 7 and 8.

As shown in figure 7, each lens is in a different position than the other lenses. for this reason, the images taken by these lenses aren't aligned with each other. Also, due to the different viewing angles of each lens relative to a feature, the error due to the height difference in each image will be different from other images, which would result in a high-altitude features error in low altitude imaging, after registration.

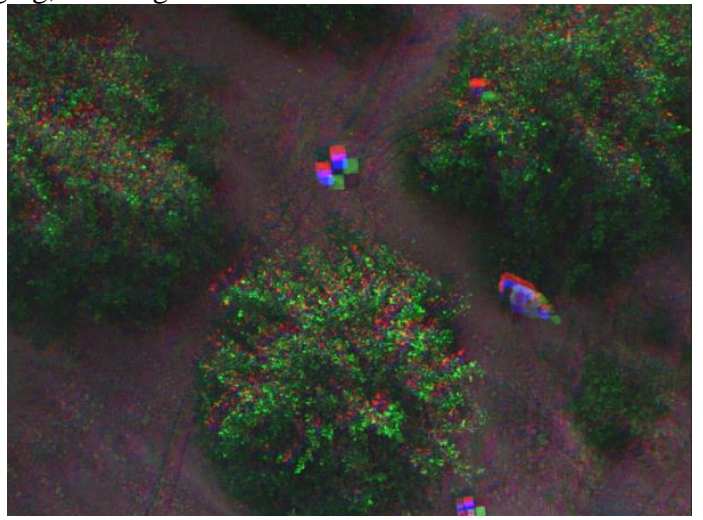

Figure 6. Miss registration in multi-spectral images
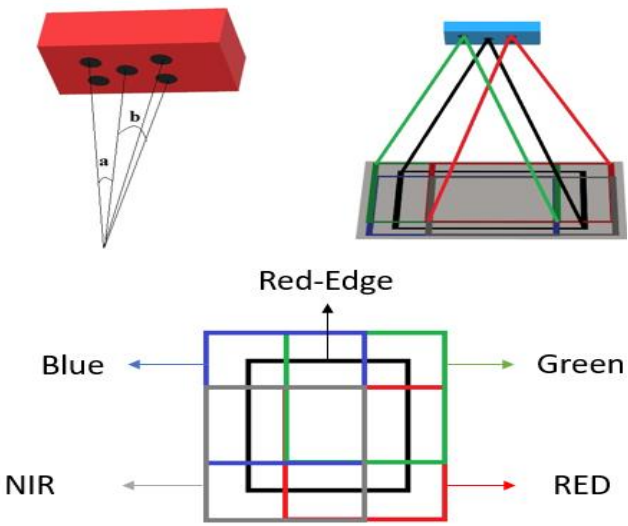

Figure 7. Lens structure and not alignement 
Since the effects of varying heights cause problems in the distribution of points, they cause the occurrence of large errors in high difference features compared to low difference features. For example, the miss-registration error occurred in a high relief area is up to 10 pixels (Fig. 8).

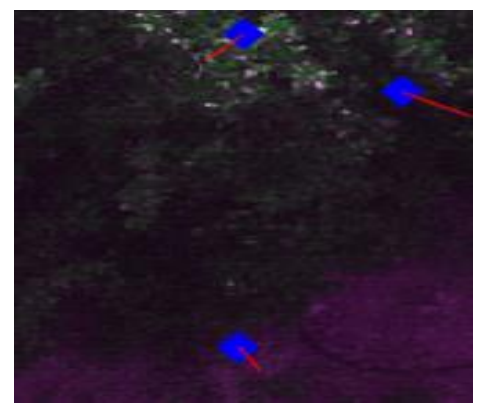

Figure 8. miss-registration error vecor in data set 3 , with $20 \mathrm{x}$ magnification and the points are presented in blue and the error vector in red lines.

In figure 8 , which is a slice of the data set 3 , it is well presented that the error vector for the points on top of the trees is larger than the error vector for the points on the ground.

Since height difference displacement is one of the important issue in the photogrammetry and the main challenge in multi-spectral cameras installed on UAVs, then an explanation about the height difference displacement will be initially given. The height difference displacement is the radial distance between a feature in the image and its correct position in the planer coordinate system relative to the center of the image (Sunita Devi, Veena, 2014). Figure 9 shows the height displacement.

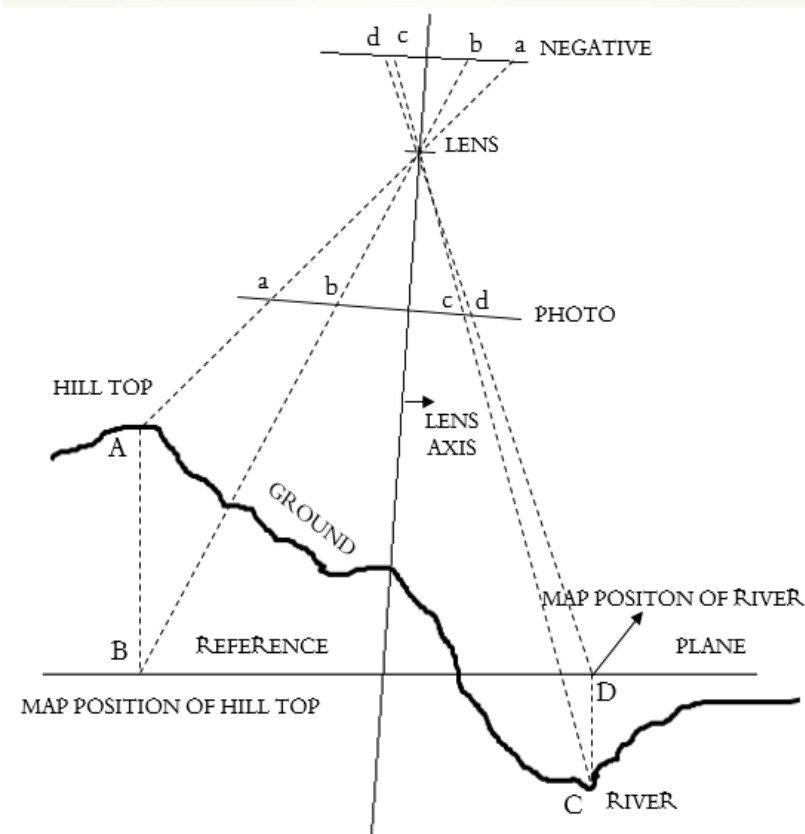

Figure 9. Relief displacement image (Sunita Devi, Veena, 2014)

This displacement is different for different heights. On the other hand, the relationship between parallax and the height of features can be expressed in Equation 1.

$$
d h=\frac{d p}{d p+b} \times H
$$

where $\quad \mathrm{dh}=$ features height

$\mathrm{dp}=$ difference of parallax

$\mathrm{b}=$ Distance between shooting stations on the photo

$\mathrm{H}=$ flight height

If the multi-spectral camera bands registered correctly to each other, the height difference displacement value can be equal to the parallax in equation 1 . Thus, with a constant flight height, the higher the difference of height of the features, the greater the displacement. Because of the need to fly at low altitude in plant degradation and plant disease applications, the data can be divided into the following three categories.

- Data with almost flat features that can be neglected due to relief displacement.

- Data with moderate heights of 1 to 3 meters, with a lack of relief displacement.

- Data with features of over 3 meters, which is important due to the relief displacement.

So, first, the data is analyzed in terms of elevation features. Then three registration methods will be discussed. In the first method, a global registration is made on the whole image. In the second method, images are divided into several patches, and in each patch feature points are extracted. According to Table 1, which shows the number of extracted and matched points, along with running time in 6,12 , and 20 patches. Therefore, if the number of patches get larger, then the more corresponding points will be added. But by according to running time, 6 patches have better results than 12 or 20 . So in this study to avoid creating gaps in the image, 6 patches have been chosen.

\begin{tabular}{|c|c|c|c|c|}
\hline $\begin{array}{c}\text { Num. } \\
\text { of } \\
\text { patches }\end{array}$ & Bands & Points & $\begin{array}{c}\text { Matched } \\
\text { points }\end{array}$ & Time (s) \\
\hline \multirow{4}{*}{6} & Red & 475 & 425 & $18 / 34$ \\
\cline { 2 - 5 } & Green & 2942 & 2888 & $18 / 22$ \\
\cline { 2 - 5 } & Blue & 691 & 645 & $17 / 51$ \\
\cline { 2 - 5 } & NIR & 1888 & 1779 & $15 / 10$ \\
\hline \multirow{4}{*}{12} & Red & 759 & 490 & $32 / 05$ \\
\cline { 2 - 5 } & Green & 3167 & 2896 & $31 / 36$ \\
\cline { 2 - 5 } & Blue & 819 & 710 & $31 / 21$ \\
\cline { 2 - 5 } & NIR & 1937 & 1791 & $29 / 49$ \\
\hline \multirow{3}{*}{20} & Red & 920 & 564 & $65 / 09$ \\
\cline { 2 - 5 } & Green & 3432 & 2924 & $69 / 50$ \\
\cline { 2 - 5 } & Blue & 1002 & 806 & $70 / 39$ \\
\cline { 2 - 5 } & NIR & 2432 & 1926 & $62 / 48$ \\
\hline
\end{tabular}

Table 1. Time, points, and matched points in different patches of data with high altitudes

In the third method, the images are divided into several patches, and after that points are extracted in each patch. Moreover, in the form of windows to the center of each point, new points are extracted, and then registration is done. This helps in distributing points appropriately throughout the image. Since the features in each area are different in height, distribution of points will be 
accompanied by problems that aggravate relief displacement. The processing steps of the method are shown in Figure 10.

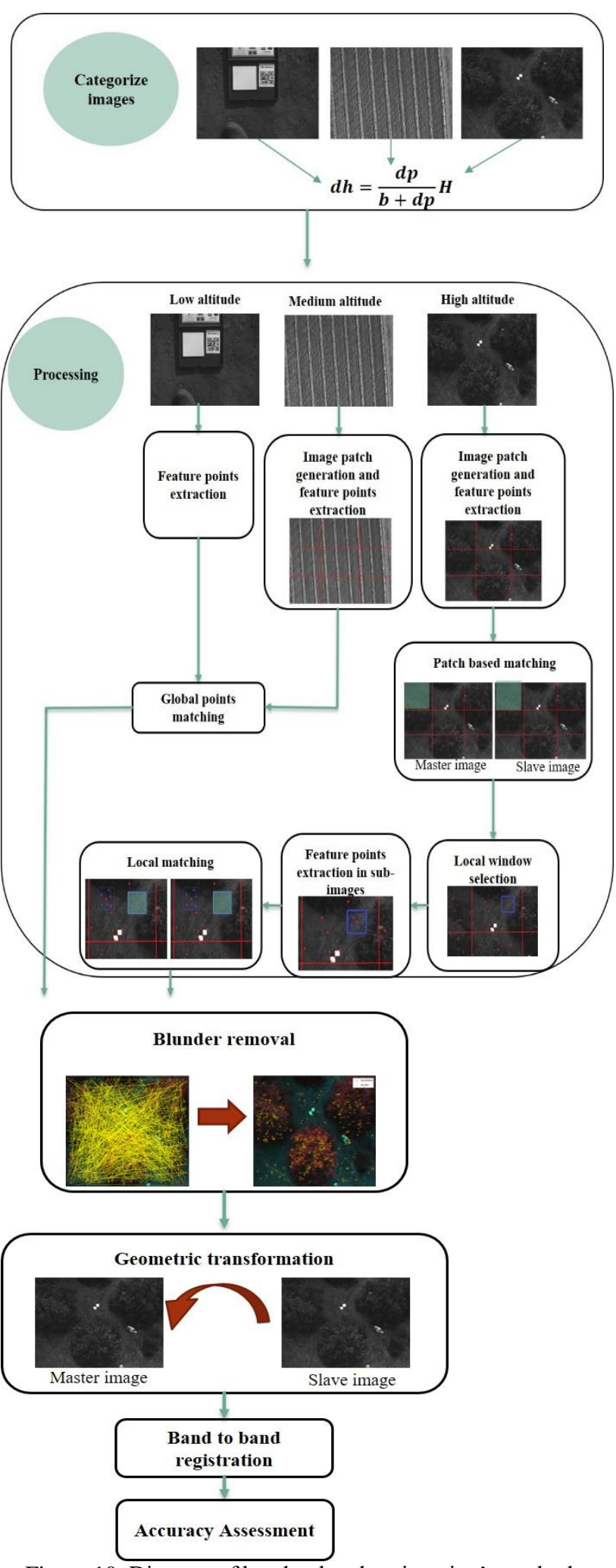

Figure 10. Diagram of band to band registration's methods.

\subsubsection{Global Registration's Method}

By using a points extraction algorithm, some points are extracted in both reference and slave images. Then the nearest neighbor ratio (NNR) or the sum of square differences (SSD) are used in order to find matched pairs. Extracted matched points are struggling with mismatches. So, points that have been mistakenly matched are eliminated using RANSAC. Finally, using a transfer function such as affine, geometric transformation between the images are performed, and a number of check points are considered and compared to control the work.

\subsubsection{Patch-Wise Registration's Method}

In this method, at first the images are divided into six overlapping patches due to the reason given in table 1 . This overlapping is due to avoid creating gaps between the patches. In each patch of the master (reference) image and other bands, the points are xtracted. In this study, 6 patches were used. After the extraction of points in each patch, the points are put together in the original image and the matching process is done. Corresponding points may be wrong, to delete the wrong points RANSAC is applied. Finally, a geometric transformation is performed using affine transfer function. Check points are also used to estimate accuracy.

\subsubsection{Patch-Wise and Local Window Registration' Method}

This method includes the following steps:

Image Patch Generation. Images are divided into six patches that are overlapped with each other, so that there is no gap between them. The number of patches is selected according to the size of the image and based on practical tests. Whatever the differences between the altitudes is high, more patches must be selected and in smaller size, but they should not be so small to complicate the computational process.

Patch based matching. The corresponding patches are considered in the reference and slave image and the feature points are extracted. Then, the matching operation is performed for each corresponding patches.

Local window selection. In the master image and the slave image, extracted points are selected as window centers. The size of these new windows should be the same everywhere.

Feature points extraction in sub-images. In this section, in each of the extracted windows (sub-images), feature points are extracted.

Local matching. In each corresponding sub-images (windows), the matching operations are performed for extracted features. This not only improves the search speed, but also results in more uniform distribution and higher density of extracted corresponding points in all parts of the image.

Blunder removal. Despite the matching process of the points, there will still be miss match points in the image. Therefore, by estimating an affine transformation model by RANSAC, the elimination of miss match points is considered. This algorithm first estimates an approximate Affine model with three random points. Then, the transformation model is fitted to all points by a fitness function.

By default, the number of maximum random trails to find inlier matches of 1000 and the confidence of finding the maximum number of inlier matches is set to 0.99 . Therefore, the maximum distance in pixels from a point is set to 1.5 for the estimation of transformation (Zhao, et al, 2018).

Geometric transformation. Considering the extracted and matched points as corresponding points, and using affine transformation model, slave images are transferred to the master image and resampled.

The transformation function defined for the coordinates of the corresponding control points in the two master images and the other image. Ttransfer, rotation, scale, or a combination of these, 
have been considered for Affine transformation. In this process, the matrix of the corresponding points in the master image is presented as $p=f(x, y, z)$ and matrix of points in slave images is presented as $q=f\left(x^{\prime}, y^{\prime}, z^{\prime}\right)$. The relation between two images is considered as $p=H . q$ where $\mathrm{H}$ is a $3 \times 3$ matrix (Yang, et al, 2016). This final relation is presented in equation 2 .

$$
\begin{gathered}
p=\left[\begin{array}{l}
x \\
y \\
z
\end{array}\right]=\left[\begin{array}{lll}
h_{11} & h_{12} & h_{13} \\
h_{21} & h_{22} & h_{23} \\
h_{31} & h_{32} & h_{33}
\end{array}\right] \cdot\left[\begin{array}{l}
x^{\prime} \\
y^{\prime} \\
z^{\prime}
\end{array}\right]= \\
{\left[\begin{array}{l}
h_{1}^{T} \\
h_{2}^{T} \\
h_{3}^{T}
\end{array}\right] \cdot\left[\begin{array}{l}
x^{\prime} \\
y^{\prime} \\
z^{\prime}
\end{array}\right]=H \cdot q}
\end{gathered}
$$

Where $\quad h_{i j}(i=1,2,3 ; j=1,2,3)$ is elements of $\mathrm{H}$ and $h_{i}(i=1,2,3)$ means $\left(h_{i 1}, h_{i 2}, h_{i 3}\right)$. In an Affine transformation $h_{31}=h_{32}=0$ and $h_{33}=1$ (Zhao, et al, 2018).

\section{RESULTS}

In the first step, comparing SIFT, SURF, Harris, FAST, and BRISK feature points extractors, the SURF operator has been selected for being applied in the proposed method due to the speed and the larger number of extracted corresponding points in comparision with other operators.

\begin{tabular}{|c|c|c|c|c|c|}
\hline Name & $\begin{array}{c}\text { Time's } \\
\text { extraction }\end{array}$ & Points & $\begin{array}{c}\text { Speed of } \\
\text { extract- } \\
\text { ion }\end{array}$ & $\begin{array}{c}\text { Match } \\
\text { Points }\end{array}$ & $\begin{array}{c}\text { Correct } \\
\text { match } \\
\text { points }\end{array}$ \\
\hline SURF & 0.864 & $\mathbf{9 6 1 8 9}$ & 89.82 & $\mathbf{2 1 5 6 8}$ & $\mathbf{1 0 4 2 5}$ \\
\hline FAST & $\mathbf{0 . 0 2}$ & 7598 & 26.32 & 981 & 491 \\
\hline SIFT & 0.823 & 90884 & $\mathbf{9 0 . 5 5}$ & 19125 & 956 \\
\hline BRISK & 0.267 & 5197 & 51.37 & 189 & 75 \\
\hline Harris & 0.259 & 2548 & 10.16 & 909 & 83 \\
\hline
\end{tabular}

Table 2. Comparison of point extraction algorithms, data set 1

\begin{tabular}{|c|c|c|c|c|c|}
\hline Name & $\begin{array}{c}\text { Time's } \\
\text { extract- } \\
\text { ion }\end{array}$ & Points & $\begin{array}{c}\text { Speed of } \\
\text { extract- } \\
\text { ion }\end{array}$ & $\begin{array}{c}\text { Match } \\
\text { points }\end{array}$ & $\begin{array}{c}\text { Correct } \\
\text { match } \\
\text { points }\end{array}$ \\
\hline SURF & 0.752 & $\mathbf{5 4 5 9 3}$ & 13.77 & $\mathbf{2 6 4 3}$ & $\mathbf{9 6 7}$ \\
\hline FAST & $\mathbf{0 . 0 2}$ & 4925 & 40.60 & 739 & 247 \\
\hline SIFT & 0.617 & 41265 & 14.95 & 1946 & 762 \\
\hline BRISK & 0.249 & 3641 & $\mathbf{6 8 . 3 8}$ & 106 & 73 \\
\hline Harris & 0.251 & 1374 & 18.26 & 742 & 79 \\
\hline
\end{tabular}

Table 3. Comparison of point extraction algorithms, data set 2

\begin{tabular}{|c|c|c|c|c|c|}
\hline Name & $\begin{array}{c}\text { Time's } \\
\text { extract-ion }\end{array}$ & Points & $\begin{array}{c}\text { Speed of } \\
\text { extract-ion }\end{array}$ & $\begin{array}{c}\text { Match } \\
\text { points }\end{array}$ & $\begin{array}{c}\text { Correct } \\
\text { match } \\
\text { points }\end{array}$ \\
\hline SURF & 0.535 & $\mathbf{2 8 2 6 5}$ & 18.926 & $\mathbf{1 0 6 6}$ & $\mathbf{2 4 3}$ \\
\hline FAST & $\mathbf{0 . 0 1}$ & 1423 & 70.27 & 361 & 55 \\
\hline SIFT & 0.623 & 20465 & 30.44 & 967 & 198 \\
\hline BRISK & 0.171 & 2084 & $\mathbf{8 2 . 0 5}$ & 95 & 53 \\
\hline Harris & 0.157 & 825 & 19.03 & 544 & 58 \\
\hline
\end{tabular}

Table 3. Comparison of point extraction algorithms, data set 3

According to Tables 2-4, the SURF algorithm has higher speed and is more precise in matching. However, the number of corresponding points must also be considered and increased.

On all the three different data, orchards contents with high difference in altitudes, agriculture with medium height differences and flat area, proposed registration strategy is performed.

In figure 11 to 13 , the distribution of extracted points for three data set based on proposed method is presented.

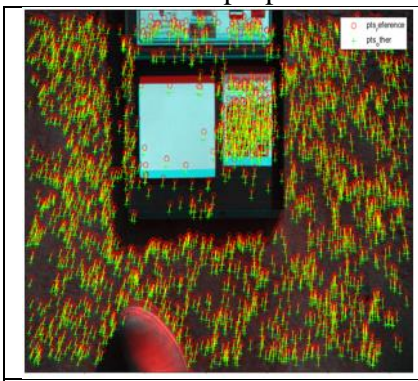

a) Matched points before registration

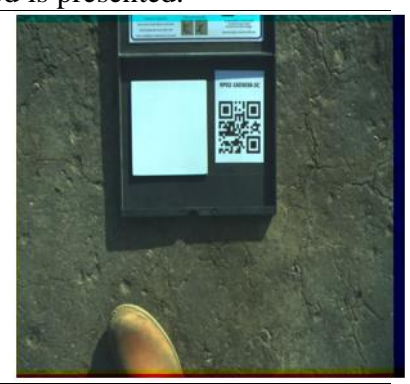

b) Registered images
Figure 11. Matching and registration, data set 1

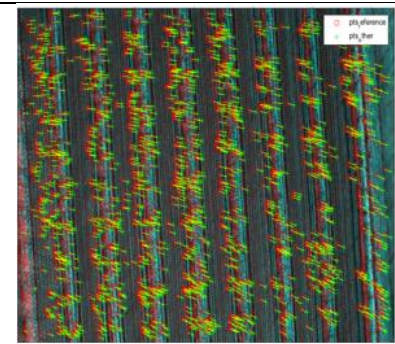

a) Matched points in global method before registration

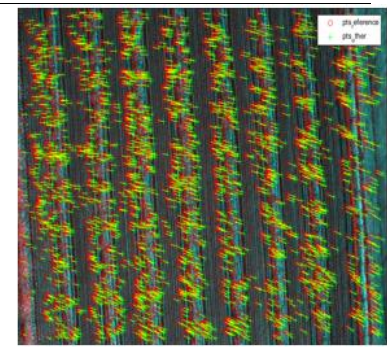

b) Matched points in pathwise method before registration

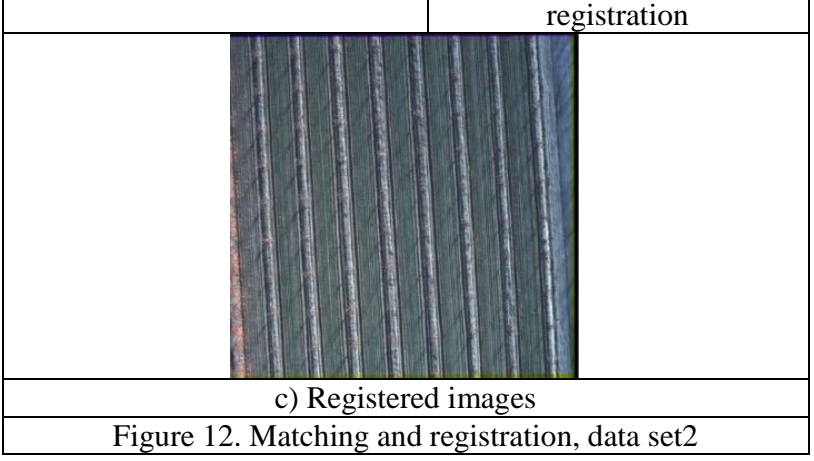



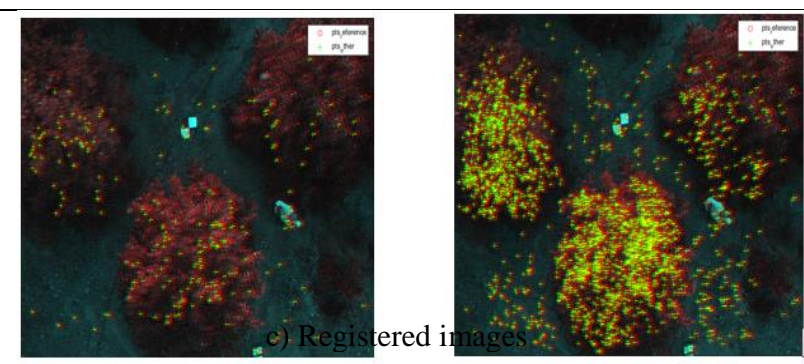

a) Matched points in global method before registration

b) Matched points in pathwise and local window method before registration

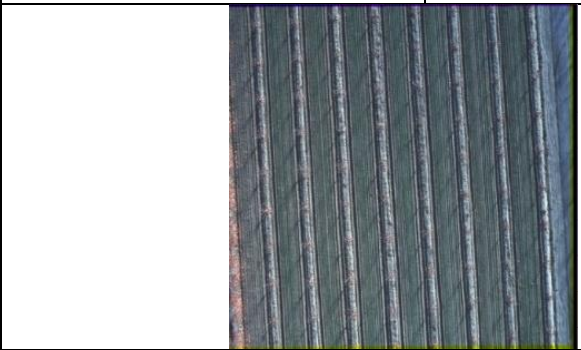

c) Registered images

Figure 12. Matching and registration, data set3

Also in figures 14 to 16 , accuracy assessment of proposed method for three data sets is displayed based on RMSE. As shown in Figures 14 to16, the accuracy of the Patch-Wise and local window method is better than the other two methods, but in the Patch-Wise and local window method, due to the higher number of extracted points in the first and second data, the processing time is considerably high. Also, for the first data, the Patch-Wise method extracts more points and increases the processing time, but this method will have an acceptable accuracy by extracting a suitable number of points and spending a suitable time for the second data. for data set 1, the Global method is very acceptable. because the number of extraction points and spending time for this data is acceptable. But for the third data which is high in height difference, the Global and Patch-Wise methods are not suitable because of the lack of uniform distribution throughout the image, and the proposed method has shown much better results.

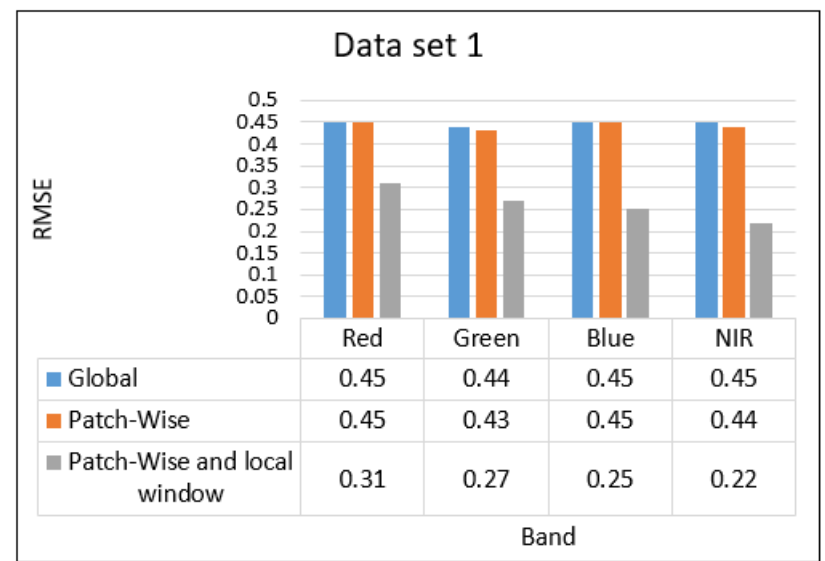

Figure 14. Final accuracy of band to band registration of the 4 bands with the reference of the red-edge band, data set 1

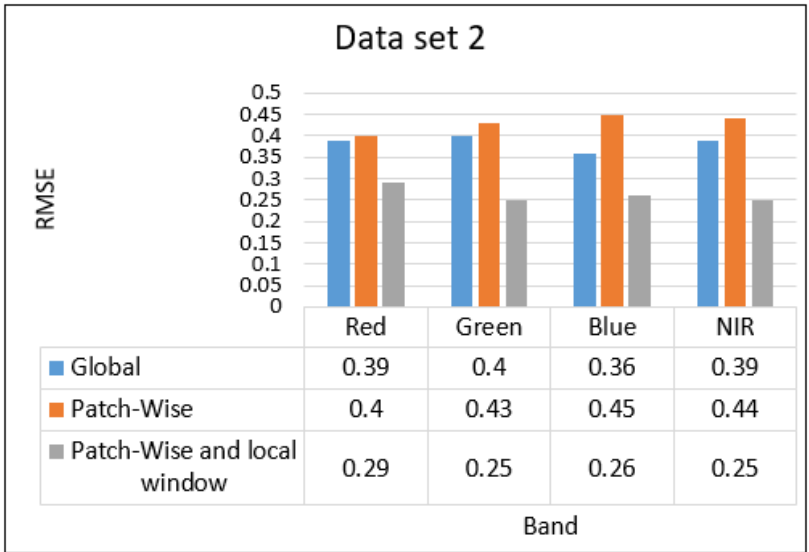

Figure 15. Final accuracy of band to band registration of the 4 bands with the reference of the red-edge band, data set 2

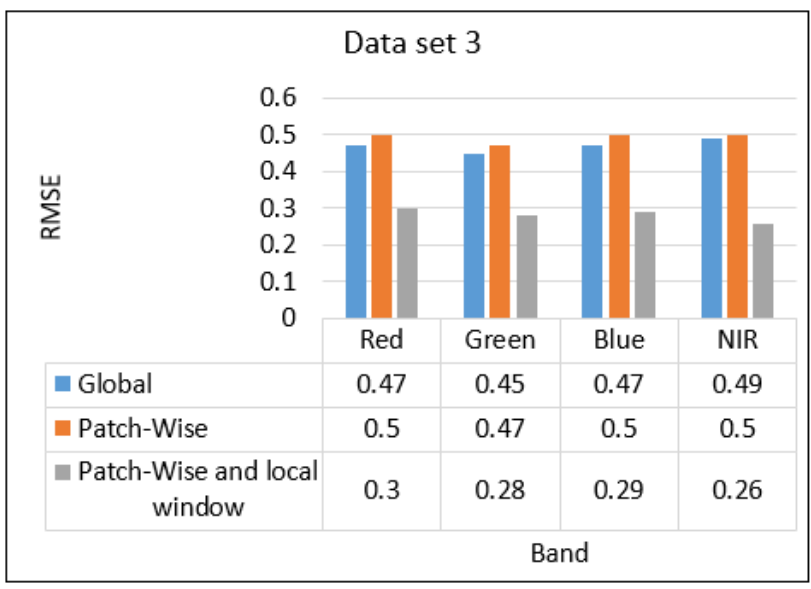

Figure 16. Final accuracy of band to band registration of the 4

bands with the reference of the red-edge band, data set 3

For Patch-Wise and local window based methods, the selection of window size is one of the important challenges. Figure 17 shows the number of extracted points for different window sizes. As the window size increases, the number of extracted points also increases, but duplicate points may be extracted, which itself increases the processing time. The larger the size of the window, the lower the correct matching rate, and the speed of the run with a sharp drop. Therefore, the window with small dimensions has more precision in matching (Zhao, et al, 2018).

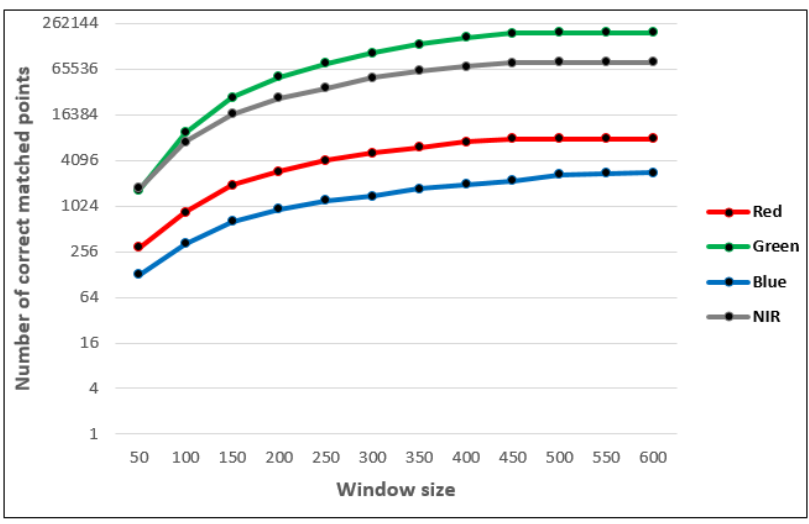

Figure 17. The number of correct matched points based on the window size 


\section{DISCUSSION AND CONCLUSION}

In this paper, effect of relief displacement on band to band registration error is investigated and a three view strategy is proposed. For evaluation of the proposed method, three different data sets from Red-Edge's multi spectral camera (MicaSense, Inc., USA) is applied.

According to achieved results, it can be concluded that missregistration error in data sets where the relief displacement with respect to flight height is high, is prominent. In these situation and based on the lower level of textural pattern in UAV based multi spectral images, common feature extractor and matching operator cannot do well. This is why the number and distribution of extracted points and consequently the accuracy of registration would not be acceptable.

Achieved results obviously present that using the proposed Patch-Wise and local window based strategy, not only the feature extraction stage but also the accuracy of mapping process is improved up to the average 0.28 pixel.

To evaluate results, in ENVI and ArcGIS software, registration process is also performed with reference of red-edge band. Also in ENVI software, it requires at least 3 points for automatic registration. The number of correct matched points for different methods are presented in figure 18 .

Results also presented that the accuracy of registration process in the proposed method is better than those from ENVI and ArcGIS software.

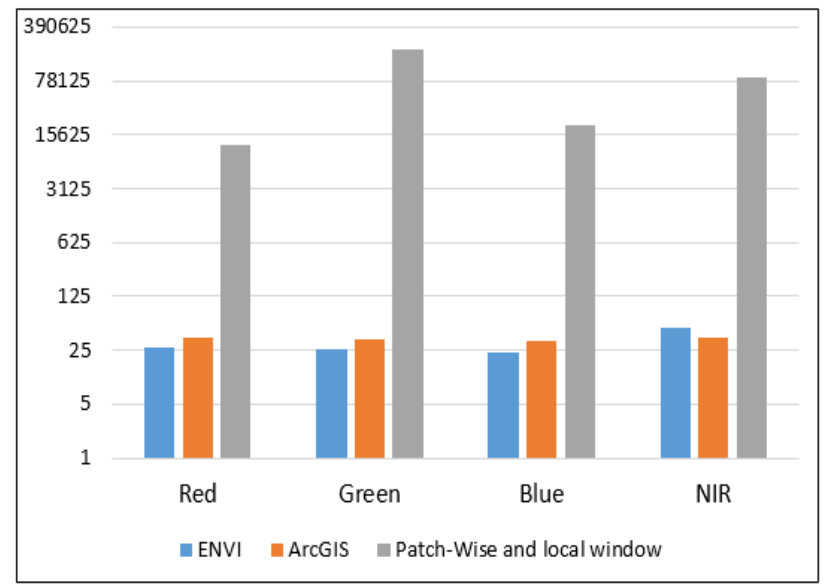

Figure 18. Comparison of the number of correct matched points between Patch-Wise and local window strategy with ENVI and ArcGIS software

As shown in figure 18, the corresponding points in the PatchWise and local window method are much higher than ENVI and ArcGIS. The corresponding points for all three categories of data have occurred in ENVI and ArcGIS software in homogeneous regions that may not be accurate. The accuracy of Patch-Wise and local window method is good and acceptable. According to figure 19 , the accuracy of the proposed method is better than ENVI and ArcGIS.

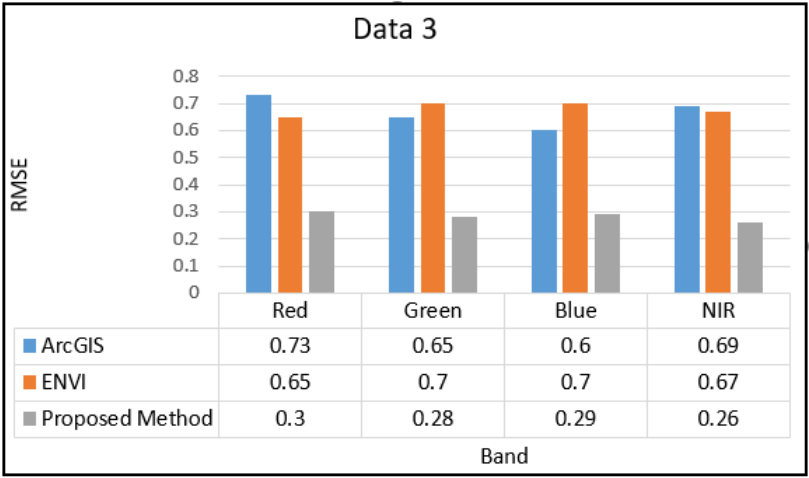

Figure 19. Compare the precision of the proposed method with the ENVI and ArcGIS software.

\section{REFERENCES}

Aicardi, I., Nex, F., Gerke, M. and Lingua, A., 2016. An imagebased approach for the co-registration of multi-temporal UAV image datasets. Remote sensing, 8(9), p.779.

Chen, Y., Zhang, X., Zhang, Y., Maybank, S.J. and Fu, Z., 2018. Visible and infrared image registration based on region features and edginess. Machine Vision and Applications, 29(1), pp.113123.

Devi, S., 2014. Veena. Measurement of Relief Displacement from Vertical Photograph. Int. J. Sci. Eng. Technol Res, 3, pp.2800-2805.

Grant, B.G., 2017, May. UAV imagery analysis: challenges and opportunities. In Long-Range Imaging II (Vol. 10204, p. 1020406). International Society for Optics and Photonics.

Gan, H., Lee, W.S. and Alchanatis, V., 2018. A photogrammetrybased image registration method for multi-camera systems-With applications in images of a tree crop. Biosystems engineering, 174, pp.89-106.

Jhan, J.P., Rau, J.Y. and Huang, C.Y., 2016. Band-to-band registration and ortho-rectification of multilens/multispectral imagery: A case study of MiniMCA-12 acquired by a fixed-wing UAS. ISPRS Journal of Photogrammetry and Remote Sensing, 114, pp.66-77.

Jhan, J.P., Rau, J.Y., Haala, N. and Cramer, M., 2017. Investigation of parallax issues for multi-lens multispectral camera band co-registration. The International Archives of Photogrammetry, Remote Sensing and Spatial Information Sciences, 42, p.157.

Jhan, J.P., Rau, J.Y. and Haala, N., 2018. Robust and adaptive band-to-band image transform of UAS miniature multi-lens multispectral camera. ISPRS Journal of Photogrammetry and Remote Sensing, 137, pp.47-60.

https://www.micasense.com/rededge-mx-sample-data

Pritt, M. and Gribbons, M.A., Lockheed Martin Corp, 2014. Automated registration of synthetic aperture radar imagery with high resolution digital elevation models. U.S. Patent 8,842,036.

Xu, F., Yu, H., Wang, J. and Yang, W., 2018, July. Accurate Registration of Multitemporal UAV Images Based on Detection 
of Major Changes. In 201821 st International Conference on Information Fusion (FUSION) (pp. 1480-1485). IEEE.

Yang, K., Tang, L., Liu, X., Dingxiang, W.U., Bian, Y., Zhenglong, L.I., 2016. Differen source image registration method based on texture common factor. Comput. Eng., Vol. 42, pp. 233-237.

Zhang, J., Yang, C., Song, H., Hoffmann, W., Zhang, D. and Zhang, G., 2016. Evaluation of an airborne remote sensing platform consisting of two consumer-grade cameras for crop identification. Remote Sensing, 8(3), p.257.

Zhao, X., Zhang, J., Yang, C., Song, H., Shi, Y., Zhou, X., Zhang, D. and Zhang, G., 2018. Registration for Optical Multimodal Remote Sensing Images Based on FAST Detection, Window Selection, and Histogram Specification. Remote Sensing, 10(5), p.663. 